\title{
Local Fluctuation of the Spectrum of a Multidimensional Anderson Tight Binding Model
}

\author{
Nariyuki Minami \\ Institute of Mathematics, University of Tsukuba, Tsukuba, Ibaraki, 305 Japan. \\ E-mail: minami@sakura.cc.tsukuba.ac.jp
}

Received: 1 April 1995

\begin{abstract}
We consider the Anderson tight binding model $H=-\Delta+V$ acting in $l^{2}\left(\mathbf{Z}^{d}\right)$ and its restriction $H^{\Lambda}$ to finite hypercubes $\Lambda \subset \mathbf{Z}^{d}$. Here $V=\left\{V_{x} ; x \in \mathbf{Z}^{d}\right\}$ is a random potential consisting of independent identically distributed random variables. Let $\left\{E_{j}(\Lambda)\right\}_{j}$ be the eigenvalues of $H^{\Lambda}$, and let $\xi_{j}(\Lambda, E)=|\Lambda|\left(E_{j}(\Lambda)-E\right)$, $j \geqq 1$, be its rescaled eigenvalues. Then assuming that the exponential decay of the fractional moment of the Green function holds for complex energies near $E$ and that the density of states $n(E)$ exists at $E$, we shall prove that the random sequence $\left\{\xi_{j}(\Lambda, E)\right\}_{j}$, considered as a point process on $\mathbf{R}^{1}$, converges weakly to the stationary Poisson point process with intensity measure $n(E) d x$ as $\Lambda$ gets large, thus extending the result of Molchanov proved for a one-dimensional continuum random Schrödinger operator. On the other hand, the exponential decay of the fractional moment of the Green function was established recently by Aizenman, Molchanov and Graf as a technical lemma for proving Anderson localization at large disorder or at extreme energy. Thus our result in this paper can be summarized as follows: near the energy $E$ where Anderson localization is expected, there is no correlation between eigenvalues of $H^{\Lambda}$ if $\Lambda$ is large.
\end{abstract}

\section{Introduction}

In this paper, we treat the multi-dimensional Anderson tight binding model, namely the discretized Schrödinger operator $H$ with a random potential $V$

$$
H=-\Delta+V
$$

acting in $l^{2}\left(\mathbf{Z}^{d}\right)$, where $\Delta$ is the discrete Laplacian defined by

$$
(\Delta u)(x)=\sum_{|y-x|=1} u(y) .
$$

We also consider the restriction $H^{\Lambda}$ of $H$ under the Dirichlet boundary condition to finite hypercubes $\Lambda \subset \mathbf{Z}^{d}$,

$$
H^{\Lambda}=\chi_{\Lambda} H \chi_{\Lambda}
$$


where $\chi_{\Lambda}$ is the multiplication by the indicator function of $\Lambda$. In the following, we assume that the random potential $V=\left\{V_{x} ; x \in \mathbf{Z}^{d}\right\}$ consists of independent, identically distributed random variables and that their common distribution has a bounded density, namely we assume

$$
P\left(V_{x} \in d v\right)=\rho(v) d v,
$$

with

$$
\|\rho\|_{\infty}<\infty
$$

Let

$$
E_{1}(\Lambda) \leqq \cdots \leqq E_{|\Lambda|}(\Lambda)
$$

be the eigenvalues of $H^{\Lambda}$. Since $V=\left\{V_{x}\right\}_{x}$ is an ergodic random field, for almost all realizations of $V$, the limit

$$
N(E)=\lim _{\Lambda \uparrow \mathbf{Z}^{d}} \frac{1}{|\Lambda|} \#\left\{j \mid E_{j}(\Lambda) \leqq E\right\}
$$

exists for all $E \in \mathbf{R}$ (see [C-L]). This limit is called the integrated density of states. Since $N(E)$ is a non-decreasing function of $E$, it is differentiable almost everywhere, and if its derivative $n(E)=d N(E) / d E$ exists at $E \in \mathbf{R}$, we call it the density of states at the energy $E$. Actually under our assumption $(1.4)$ and $(1.5), N(\cdot)$ is known to be absolutely continuous, so that (1.7) tells us that average spacing of eigenvalues is of order of $|\Lambda|^{-1}$ near the energy $E$ where the density of states $n(E)$ exists and is positive. A natural question then arises as to measuring the local fluctuation of the spectrum, namely one will be interested in the limiting probability distribution of the rescaled spectrum

$$
\xi_{j}(\Lambda ; E)=|\Lambda|\left(E_{j}(\Lambda)-E\right), \quad j=1, \ldots,|\Lambda|,
$$

as $\Lambda$ gets large. To give a concise formulation of the problem, it is appropriate to use the notion of point process and their weak convergence.

Let $\mathscr{M}\left(\mathbf{R}^{1}\right)$ be the space of all non-negative Radon measures on $\mathbf{R}^{1}$. We say that a sequence $\left\{m_{n}\right\}_{n}$ in $\mathscr{M}\left(\mathbf{R}^{1}\right)$ converges to $m \in \mathscr{M}\left(\mathbf{R}^{1}\right)$ vaguely if

$$
\lim _{n \rightarrow \infty} \int \varphi(x) m_{n}(d x)=\int \varphi(x) m(d x)
$$

holds for all $\varphi$ which belong to $C_{K}^{+}\left(\mathbf{R}^{1}\right)$, the space of all non-negative continuous functions with compact support.

This convergence concept defines a topology on $\mathscr{M}\left(\mathbf{R}^{1}\right)$ which is called the vague topology. Let further $\mathscr{M}_{p}\left(\mathbf{R}^{1}\right)$ be the subspace of $\mathscr{M}\left(\mathbf{R}^{1}\right)$ consisting of all integer valued Radon measures on $\mathbf{R}^{1}$. Each $\xi \in \mathscr{M}_{p}\left(\mathbf{R}^{1}\right)$ can be written as

$$
\xi(d x)=\sum_{j} \delta_{\xi_{j}}(d x)
$$

with a sequence $\left\{\xi_{j}\right\}$ having no finite accumulation point. It is known that $\mathscr{M}_{p}\left(\mathbf{R}^{1}\right)$ is closed in $\mathscr{M}\left(\mathbf{R}^{1}\right)$ with respect to the vague topology. A random variable $\xi^{\omega}$ taking values in $\mathscr{M}_{p}\left(\mathbf{R}^{1}\right)$ is called a point process. We say $\mu(d x)$ is the intensity measure of $\xi^{\omega}$ if

$$
\mu(A)=E\left[\xi^{\omega}(A)\right]
$$

for each Borel set $A$. 
Now consider a sequence $\left\{\xi_{n}^{\omega}\right\}_{n}$ of point processes defined on a probability space $(\Omega, \mathscr{F}, \mathbf{P})$. This sequence converges weakly to a point process $\xi^{\bar{\omega}}$ defined on a (possibly different) probability space $(\bar{\Omega}, \overline{\mathscr{F}}, \overline{\mathbf{P}})$ iff, by definition, for any bounded continuous function $\Phi(\xi)$ on $\mathscr{M}_{p}\left(\mathbf{R}^{1}\right)$ one has

$$
\lim _{n \rightarrow \infty} \int \Phi\left(\xi_{n}^{\omega}\right) \mathbf{P}(d \omega)=\int \Phi\left(\xi^{\bar{\omega}}\right) \overline{\mathbf{P}}(d \bar{\omega})
$$

On the other hand, this abstract definition is known to be equivalent to each of the following two statements:

(1) For any $\varphi \in C_{K}^{+}\left(\mathbf{R}^{1}\right)$,

$$
\lim _{n \rightarrow \infty} \mathbf{E}_{\mathbf{P}}\left[e^{-\xi_{n}^{\omega}(\varphi)}\right]=\mathbf{E}_{\overline{\mathbf{P}}}\left[e^{-\xi^{\bar{\omega}}(\varphi)}\right],
$$

where we have set

$$
\xi_{n}^{\omega}(\varphi)=\int \varphi(x) \xi_{n}(d x), \text { etc. }
$$

(2) For any $l \geqq 1, k_{j} \geqq 0$, and disjoint intervals $I_{j}, j=1, \ldots, l$, such that

$$
\overline{\mathbf{P}}\left(\xi\left(\partial I_{j}\right)>0\right)=0,
$$

one has

$$
\lim _{n \rightarrow \infty} \mathbf{P}\left(\xi_{n}\left(I_{j}\right)=k_{j}, j=1, \ldots, l\right)=\overline{\mathbf{P}}\left(\xi\left(I_{j}\right)=k_{j}, j=1, \ldots, l\right)
$$

Among elementary examples of point processes, the Poisson point process is the most important one. $\xi^{\omega}$ is said to be the Poisson point process with intensity measure $\mu(d x)$ if it satisfies the following two conditions:

(a) For each bounded Borel set $A, \xi^{\omega}(A)$ obeys the Poisson distribution with parameter $\mu(A)$, namely

$$
\mathbf{P}\left(\xi^{\omega}(A)=k\right)=e^{-\mu(A)} \frac{\mu(A)^{k}}{k !}, \quad k \geqq 0 ;
$$

(b) If $A_{1}, \ldots, A_{n}$ are disjoint, then $\xi\left(A_{1}\right), \ldots, \xi\left(A_{n}\right)$ are independent random variables.

Now we can state our result. Let $\Lambda=[1, L]^{d}, L=1,2, \ldots$, be hypercubes in $\mathbf{Z}^{d}$ and for $z$ with $\Im z>0$ let

$$
G^{\Lambda}(z ; x, y)=\left(H^{\Lambda}-z\right)^{-1}(x, y)
$$

and

$$
G(z ; x, y)=(H-z)^{-1}(x, y)
$$

be the Green functions of $H^{\Lambda}$ and $H$ respectively. Define the point process $\xi(\Lambda ; E)$ by

$$
\xi(\Lambda ; E)(d x)=\sum_{j} \delta_{|\Lambda|\left(E_{J}(\Lambda)-E\right)}(d x),
$$

where $E_{j}(\Lambda)$ are eigenvalues of $H^{\Lambda}$ as in (1.6), and $\delta_{x}$ is the Dirac measure concentrated at $x$. Then we can prove 
Theorem. Suppose that the density of states $n(E)$ exists at $E$ and is positive and that the fractional moment of the Green function decays exponentially fast in the following sense:

There are $s_{0} \in(0,1), C>0, m>0$ and $r>0$ such that

$$
\mathbf{E}\left[\left|G^{D}(z ; x, y)\right|^{s_{0}}\right] \leqq C e^{-m|x-y|}
$$

for any hypercube $D \subset \mathbf{Z}^{d}, x \in D, y \in \partial D$, and

$$
z \in\{z|\Im z>0,| z-E \mid<r\} .
$$

Here we shall say $y \in \partial D$ iff $y \in D$ and there is a $y^{\prime} \in D^{c}$ such that $\left|y-y^{\prime}\right|=1$. Under these conditions, the point process $\xi(\Lambda ; E)$ defined by (1.19) converges weakly, as $L \rightarrow \infty$, to the Poisson point process $\xi$ with intensity measure $n(E) d x$.

Remark. The following sufficient conditions for (1.20) were obtained by Aizenman, Molchanov and Graf:

i) If $\rho(x)$ is bounded and if there is a compact interval $[a, b]$ such that $\rho(x)$ is non-decreasing on $(-\infty, a]$ and is non-increasing on $[b, \infty)$, then there is an $E(\rho)$ such that (1.20) is true for $|E|>E(\rho)$ (see Sect. 3 of [A-M]);

ii) If $\|\rho\|_{\infty}$ is sufficiently small, then (1.20) holds for all $E$ (see [G]).

In case $d=1$, if we assume the finiteness of $\|\rho\|_{\infty}$ and $E\left[\left|V_{x}\right|^{\varepsilon}\right]$ for some $\varepsilon>0$, then (1.20) holds for any $E$. The proof of this fact will be given in the Appendix for the reader's convenience.

A limit theorem of this type was first proved by Molchanov ([Mo2]) for the one-dimensional random Schrödinger operator

$$
-\frac{d^{2}}{d t^{2}}+F\left(x_{t}(\omega)\right), \quad-\infty<t<\infty
$$

and its restriction to large finite intervals under the Dirichlet boundary condition. Here $\left\{x_{t}(\omega)\right\}$ is the stationary Brownian motion on a compact Riemannian manifold $K$ and $F$ is a smooth, "non-flat" function on $K$ with $\inf _{x} F(x)=0$.

The random Schrödinger operator (1.21) was the first mathematical model for which Anderson localization was rigorously established (see [G-M-P] and [Mol]). Moreover the density of states $n(E)$ exists and is positive for all $E>0$, and accordingly the convergence of $\xi(\Lambda ; E)$, where $\Lambda=[0, L]$, to the Poisson point process holds for any $E>0$.

Molchanov's result is very beautiful and physically significant, as it is one of few examples of energy level statistics which can be rigorously performed. (See however [Mi] for an elementary example of a Schrödinger operator for which a different kind of energy level statistics can be performed and an approximate Poisson statistics is obtained.) Molchanov's proof is based on some precise analysis of localization on a large finite interval which had been done in [Mol]. Although his idea was intuitively very natural and independent of the dimensionality of the space, as we shall show below, the technique he used is valid only in one-dimensional models. Since nowadays we have a fairly good understanding of localization for the Anderson tight binding model in general dimension, it is desirable to give a direct justification of the above mentioned idea to obtain a dimension-independent proof of the local Poisson nature of the spectrum. 
Now let us describe the basic idea behind our theorem. First note that the condition (1.4-5) and (1.20) imply that we have Anderson localization for $H$. Namely with probability one, the spectrum of $H$ is pure point in the vicinity of $E$, and corresponding eigenfunctions decay exponentially fast. This can be easily seen by slightly modifying the argument of Delyon, Lévy and Souillard [D-L-S]. Suppose the exponential decay of eigenfunctions holds also for $H^{\Lambda}$ when $\Lambda=[1, L]^{d}$ is large. In other words, suppose that for each eigenfunction $\psi_{E_{j}}$ of $H^{\Lambda}$ with eigenvalue $E_{j}$, which is in the vicinity of $E$, we can somehow define a "center" $X_{E_{J}}$ of localization so that $\psi_{E_{j}}(x)$ decays like $\exp \left[-\alpha\left|x-X_{E_{j}}\right|\right]$ for some $\alpha>0$. It is natural to suppose that those $X_{E_{j}}$ are distributed in $\Lambda$ "uniformly" so that if we divide $\Lambda$ into small cells $C_{p}$ of side length $\sim L^{a}, 0<a<1$, then most eigenfunctions $\psi_{E}$, would have their center inside some $C_{p}$ away from the boundary so that $\left|\psi_{E_{J}}(x)\right|$ would be very small on $\partial C_{p}$. (In fact, this is exactly what Molchanov showed for the model (1.21) and it would be a nice mathematical problem to do the same thing for the multidimensional Anderson tight binding model.) This suggests to us that as far as the spectral properties of $H^{\Lambda}$ near the energy $E$ concerns, we could approximate $H^{\Lambda}$ by the direct sum of $H^{C_{p}}$. Since $H^{C_{p}}$ are statistically independent for different $p$, this means that the spectrum of $H^{\Lambda}$ near $E$, viewed as a point process, is approximated by a superposition of independent point processes, each of which coming from $H^{C_{p}}$. Hence if we set

$$
\eta\left(C_{p} ; E\right)(d x)=\sum_{j} \delta_{|\Lambda|\left(E_{j}\left(C_{p}\right)-E\right)}(d x)
$$

$\left\{E_{j}\left(C_{p}\right)\right\}_{j}$ being the eigenvalues of $H^{C_{p}}$, then $\xi(\Lambda ; E)$ can be approximated by

$$
\eta(L ; E)=\sum_{p} \eta\left(C_{p} ; E\right)(d x)
$$

As remarked above, $\left\{\eta\left(C_{p} ; E\right)\right\}_{p}$ are independent for different $p$. On the other hand, the mean spacing of the eigenvalues $\left\{E_{j}\left(C_{p}\right)\right\}_{j}$ is of order $L^{-a d}$, so that by (1.22), each $\eta\left(C_{p} ; E\right)$ is asymptotically negligible as $L \rightarrow \infty$. (See [D-V] and the next section for the precise meaning of "negligibility.") Hence by the general theory of point processes (see Theorem 9.2.V of [D-V]), $\eta(L ; E)$, being the superposition of a uniformly asymptotically negligible array, converges weakly to the Poisson point process with intensity measure $n(E) d x$ as soon as for any bounded interval $A$, the following two conditions hold:

$$
\begin{gathered}
\sum_{p} P\left(\eta\left(C_{p} ; E\right)(A) \geqq 1\right) \rightarrow n(E)|A| ; \\
\sum_{p} P\left(\eta\left(C_{p} ; E\right)(A) \geqq 2\right) \rightarrow 0 .
\end{gathered}
$$

The justification that $\xi(\Lambda ; E)$ can be replaced by $\eta(L ; E)$ as well as the verification of the above two conditions will be given in the next section. It seems that the importance of the conditions like (1.24) and (1.25) is overlooked by most physicists. In fact, all we can tell from the fact that our $\xi(\Lambda ; E)$ can be approximated by the superposition of small independent point processes is that the limit of $\xi(\Lambda ; E)$, if any, is an infinitely divisible point process, which may be quite different from Poisson point process, and in fact it is the verification of these conditions which consists of the main and the hardest part of the proof in [Mo2]. 


\section{Proof of Theorem}

The proof breaks into several steps.

Step 1. Define the class $\mathscr{A}$ of test functions of the form

$$
f(x)=\sum_{j=1}^{n} \frac{a_{j} \tau}{\left(x-\sigma_{j}\right)^{2}+\tau^{2}}
$$

with $n \geqq 1, \tau>0$ and $a_{j}>0, \sigma_{j} \in \mathbf{R}$ for $j=1, \ldots, n$.

Lemma 1. Let $\xi_{n}, n \geqq 1$, and $\xi$ be point processes on $\mathbf{R}^{1}$ with intensity measures $\mu_{n}(d x)$ and $\mu(d x)$ respectively. Suppose there is a constant $c>0$ such that

$$
\mu_{n}(d x) \leqq c d x, \quad n \geqq 1, \mu(d x) \leqq c d x .
$$

Then the following two assertions are equivalent:

(i) $\xi_{n}$ converges weakly to $\xi$;

(ii) for any $f \in \mathscr{A}$, one has

$$
\lim _{n \rightarrow \infty} \mathbf{E}\left[\exp \left(-\xi_{n}(f)\right)\right]=\mathbf{E}[\exp (-\xi(f))]
$$

Proof. For a non-negative Borel function $\varphi$, define for $n \geqq 1$,

$$
\mathscr{L}_{n}(\varphi)=\mathbf{E}\left[\exp \left(-\xi_{n}(\varphi)\right)\right]
$$

and

$$
\mathscr{L}(\varphi)=\mathbf{E}[\exp (-\xi(\varphi))] .
$$

Then the assertion (i) is equivalent to

(i) $\mathscr{L}_{n}(\varphi) \rightarrow \mathscr{L}(\varphi)$, for any $\varphi \in C_{K}^{+}$.

Let $L_{+}^{1}$ be the totality of all non-negative elements in $L^{1}\left(\mathbf{R}^{1}\right)$. It is easily seen from our assumption (2.2) that the functionals $\mathscr{L}_{n}$ and $\mathscr{L}$ are uniformly equicontinuous on $L_{+}^{1}$. Since $C_{K}^{+}\left(\mathbf{R}^{1}\right)$ is dense in $L_{+}^{1}$, the assertion (i) $)^{\prime}$ can be extended to hold for all $\varphi \in L_{1}^{+}$, and in particular for all $\varphi \in \mathscr{A}$. Hence (i) implies (ii). To show the converse, it suffices, by the same reason, to prove that $\mathscr{A}$ is dense in $L_{1}^{+}$. For this purpose, it is sufficient to prove that for any bounded interval $(a, b)$, its indicator $1_{(a, b)}(x)$ can be approximated in the $L^{1}$-sense by elements in $\mathscr{A}$.

Now for $\tau>0$ and $n=1,2, \ldots$, define

$$
\Phi_{\tau}(x)=\frac{1}{\pi} \int_{a}^{b} \frac{\tau}{(x-\sigma)^{2}+\tau^{2}} d \sigma
$$

and

$$
\Phi_{n, \tau}(x)=\frac{1}{\pi} \sum_{j=0}^{n-1} \frac{(b-a) \tau / n}{\left(x-\sigma_{n, j}\right)^{2}+\tau^{2}},
$$

where

$$
\sigma_{j}^{n}=a+\frac{(b-a) j}{n}
$$

We shall show that

$$
\lim _{n \rightarrow \infty} \Phi_{n, \tau}=\Phi_{\tau}
$$


and

$$
\lim _{\tau \downarrow 0} \Phi_{\tau}=1_{(a, b)}
$$

hold both in $L^{1}$-sense.

To show (2.9), set

$$
\Psi(x, \sigma)=\frac{\left(\sigma-\sigma_{j}^{n}\right)\left(2 x-\sigma-\sigma_{j}^{n}\right)}{\left\{(x-\sigma)^{2}+\tau^{2}\right\}\left\{\left(x-\sigma_{j}^{n}\right)^{2}+\tau^{2}\right\}},
$$

where $\sigma \in[a, b)$ and $\sigma_{j}^{n}$ is chosen so that $\sigma \in\left[\sigma_{j}^{n}, \sigma_{j+1}^{n}\right)$. Then for some constant $C=C_{\tau}>0$, we have

$$
|\Psi(x, \sigma)| \leqq C \frac{|x|}{n\left(1+x^{2}\right)^{2}}, \quad \text { for } x \notin[a-1, b+1]
$$

and

$$
|\Psi(x, \sigma)| \leqq C \frac{1}{n}, \quad \text { for } x \in[a-1, b+1]
$$

so that

$$
\int_{-\infty}^{\infty}\left|\Phi_{\tau}(x)-\Phi_{n, \tau}(x)\right| d x \leqq \frac{\tau}{\pi} \int_{a}^{b} d \sigma \int_{-\infty}^{\infty} d x|\Psi(x, \sigma)|=O\left(n^{-1}\right) .
$$

To show (2.10), note that

$$
\Phi_{\tau}(x)=\frac{1}{\pi}\left[\tan ^{-1} y\right]_{(a-x) / \tau}^{(b-x) / \tau} .
$$

Hence if $a<x<b$, we have $0<\Phi_{\tau}(x)<1$ and $\Phi_{\tau}(x) \uparrow 1$ as $\tau \downarrow 0$. On the other hand,

$$
\int_{-\infty}^{\infty} \Phi_{\tau}(x) d x=b-a=\int_{-\infty}^{\infty} 1_{(a, b)}(x) d x
$$

From these considerations, it is easy to conclude (2.10).

Step 2. Let $\xi$ be the Poisson point process on $\mathbf{R}^{1}$ with intensity measure $\mu(d x)=$ $n(E) d x$. Let us verify that this $\xi$ and $\xi(\Lambda ; E)$ defined by (1.19) satisfy the conditions of Lemma 1.

To this end, let

$$
f_{\zeta}(x)=\frac{\tau}{(x-\sigma)^{2}+\tau^{2}}
$$

for arbitrary $\zeta=\sigma+i \tau \in \mathbf{C}_{+}$, where $\mathbf{C}_{+}$is the open upper half plane. Then

$$
\begin{aligned}
\mathbf{E}\left[\xi(\Lambda ; E)\left(f_{\zeta}\right)\right] & =\mathbf{E}\left[\sum_{j} \frac{\tau}{\left\{|\Lambda|\left(E_{j}(\Lambda)-E\right)-\sigma\right\}^{2}+\tau^{2}}\right] \\
& =\frac{1}{|\Lambda|} \mathbf{E}\left[\sum_{j} \frac{|\Lambda|^{-1} \tau}{\left(E_{j}(\Lambda)-E-|\Lambda|^{-1} \sigma\right)^{2}+\left(|\Lambda|^{-1} \tau\right)^{2}}\right] \\
& =\frac{1}{|\Lambda|} \mathbf{E}\left[\operatorname{Tr} \Im G^{\Lambda}\left(E+|\Lambda|^{-1} \zeta\right)\right] \\
& =\frac{1}{|\Lambda|} \sum_{x \in \Lambda} \mathbf{E}\left[\Im G^{\Lambda}\left(E+|\Lambda|^{-1} \zeta ; x, x\right)\right]
\end{aligned}
$$


If we construct for $z \in \mathbf{C}_{+}$

$$
\hat{G}^{\Lambda}(z)=\left(-H^{\Lambda}-V_{x} \delta_{x}-z\right)^{-1},
$$

where $\delta_{x} \in l^{2}\left(Z^{d}\right)$ is defined by $\delta_{x}(y)=\delta_{x y}$, then by the resolvent equation

$$
G^{\Lambda}\left(E+|\Lambda|^{-1} \zeta ; x, x\right)=\frac{1}{V_{x}-\Gamma}
$$

where

$$
\Gamma=-\hat{G}^{\Lambda}\left(E+|\Lambda|^{-1} \zeta ; x, x\right)^{-1}
$$

Since $\Im \Gamma>0$, we can compute

$$
\begin{aligned}
\mathbf{E}\left[\Im G^{\Lambda}\left(E+|\Lambda|^{-1} \zeta ; x, x\right)\right] & =\mathbf{E}\left[\int_{-\infty}^{\infty} \frac{\Im \Gamma}{(v-\Re \Gamma)^{2}+(\Im \Gamma)^{2}} \rho(v) d v\right] \\
& \leqq\|\rho\|_{\infty} \mathbf{E} \int \frac{\Im \Gamma}{(v-\Re \Gamma)^{2}+(\Im \Gamma)^{2}} d v=\pi\|\rho\|_{\infty} \\
& =\|\rho\|_{\infty} \int_{-\infty}^{\infty} f_{\zeta}(x) d x
\end{aligned}
$$

This shows

$$
\mathbf{E}[\xi(\Lambda ; E)(d x)] \leqq\|\rho\|_{\infty} d x,
$$

and by Wegner's inequality (see Theorem 3.2 of $[\mathrm{S}]$ ),

$$
n(E) \leqq\|\rho\|_{\infty} \text {. }
$$

Hence according to Lemma 1 , in order to prove Theorem, it suffices to prove for any $a_{j}>0, j=1, \ldots, n$ and $\zeta_{j}=\sigma_{j}+i \tau, \sigma_{j} \in \mathbf{R}, j=1, \ldots, n, \tau>0$ that

$$
\lim _{L \rightarrow \infty} \mathbf{E}\left[\exp \left\{-\frac{1}{|\Lambda|} \sum_{j=1}^{n} a_{j} \Im \operatorname{Tr} G^{\Lambda}\left(E+|\Lambda|^{-1} \zeta_{j}\right)\right\}\right]=\mathscr{L}_{P}(\varphi)
$$

where

$$
\varphi(x)=\sum_{j=1}^{n} a_{j} \frac{\tau}{\left(x-\sigma_{j}\right)^{2}+\tau^{2}} \in \mathscr{A}
$$

and

$$
\mathscr{L}_{P}(\varphi) \equiv \mathbf{E}[\exp \{-\xi(\varphi)\}],
$$

$\xi$ being the Poisson point process with intensity measure $n(E) d x$.

Remark. Actually we have

$$
\mathscr{L}_{P}(f)=\exp \left\{n(E) \int_{-\infty}^{\infty}\left(e^{-f(x)}-1\right) d x\right\},
$$

for any non-negative integrable function $f$. But we do not need this formula explicitly. 
Step 3. Let $\left\{N_{L}\right\}$ and $\left\{l_{L}\right\}$ be increasing integer valued sequences diverging to infinity as $L \rightarrow \infty$ in such a way that

$$
N_{L}=o(L), \quad l_{L}=o\left(L / N_{L}\right) .
$$

We shall choose $N_{L}$ and $l_{L}$ more specifically later.

Now divide $(0, L]^{d} \subset \mathbf{R}^{d}$ into $N_{L}^{d}$ equal cubes $\bar{C}_{p}, p=1, \ldots, N_{L}^{d}$, with side length $L / N_{L}$ and of the form $(a, b]^{d}$, and define

$$
C_{p}=\bar{C}_{p} \cap Z^{d}
$$

and

$$
\operatorname{int}\left(C_{p}\right)=\left\{x \in C_{p} \mid \operatorname{dist}\left(x, \partial C_{p}\right)>l_{L}\right\} .
$$

For any $z \in \mathbf{C}_{+}$and $x \in \operatorname{int}\left(C_{p}\right)$, we have by the well known perturbation formula (see e.g. Sect. 4 of $[\mathrm{S}]$ ),

$$
G^{\Lambda}(z ; x, x)=G^{C_{p}}(z ; x, x)+\sum_{\left\langle y, y^{\prime}\right\rangle \in \partial C_{p}} G^{C_{p}}(z ; x, y) G^{\Lambda}\left(z ; y^{\prime}, x\right)
$$

where $\left\langle y, y^{\prime}\right\rangle \in \partial C_{p}$ means that $y \in C_{p}, y^{\prime} \in \mathbf{Z}^{d} \backslash C_{p}$ and $\left|y-y^{\prime}\right|=1$. Then it is easy to see

$$
\begin{aligned}
& \left|\frac{1}{|\Lambda|} \Im \operatorname{Tr} G^{\Lambda}(z)-\frac{1}{|\Lambda|} \sum_{p} \Im \operatorname{Tr} G^{C_{p}}(z)\right| \\
& \leqq \\
& \quad \frac{1}{|\Lambda|} \sum_{p} \sum_{x \in C_{p} \backslash \operatorname{int}\left(C_{p}\right)}\left\{\Im G^{C_{p}}(z ; x, x)+\Im G^{\Lambda}(z ; x, x)\right\} \\
& \quad+\frac{1}{|\Lambda|} \sum_{p} \sum_{x \in \operatorname{int}\left(C_{p}\right)} \sum_{\left\langle y, y^{\prime}\right\rangle \in \partial C_{p}}\left|G^{C_{p}}(z ; x, y)\right|\left|G^{\Lambda}\left(z ; y^{\prime}, x\right)\right| \\
& =A_{L}+B_{L} .
\end{aligned}
$$

As in (2.22), $\mathbf{E}\left[\Im G^{C_{p}}(z ; x, x)\right]$ and $\mathbf{E}\left[\Im G^{\Lambda}(z ; x, x)\right]$ are bounded by some constant independent of $x, p, \Lambda$ and $z \in \mathbf{C}_{+}$. Hence

$$
\mathbf{E}\left[A_{L}\right]=O\left(L^{-d} N_{L}^{d}\left(L / N_{L}\right)^{d-1} l_{L}\right)=O\left(N_{L} L^{-1} l_{L}\right)
$$

as $L \rightarrow \infty$.

On the other hand, we can write

$$
\mathbf{E}\left[B_{L}^{s_{0} / 2}\right] \leqq|\Lambda|^{-s_{0} / 2} \sum_{p} \sum_{x \in \operatorname{int}\left(C_{p}\right)} \sum_{\left\langle y, y^{\prime}\right\rangle \in \partial C_{p}} \sqrt{\mathbf{E}\left|G^{C_{p}}(z ; x, y)\right|^{s_{0}}} \sqrt{\mathbf{E}\left|G^{\Lambda}\left(z ; y^{\prime}, x\right)\right|^{s_{0}}}
$$

By formula (2.22) of [A-M] or Lemma 5 of [G], $\mathbf{E}\left|G^{\Lambda}\left(z ; y^{\prime}, x\right)\right|^{s_{0}}$ is bounded by some constant independent of $\Lambda, z \in \mathbf{C}_{+}$, and $x, y^{\prime} \in \Lambda$. Hence by condition (1.20), we obtain the estimation

$$
\begin{aligned}
\mathbf{E}\left[B_{L}^{s_{0} / 2}\right] & \leqq \text { const. }|\Lambda|^{-s_{0} / 2} \sum_{p} \sum_{x \in \operatorname{int}\left(C_{p}\right)} \sum_{\left\langle y, y^{\prime}\right\rangle \in \partial C_{p}} e^{-m|x-y|} \\
& =O\left(L^{d\left(2-\frac{s_{0}}{2}\right)-1} N_{L}^{-d+1} l_{L} e^{-m l_{L}}\right) .
\end{aligned}
$$


If we take $N_{L}=L^{\alpha}$ with $0<\alpha<1$ and $l_{L}=\beta \ln L$ with sufficiently large $\beta$, say

$$
\beta>\frac{1}{m}\left\{d\left(2-\frac{s_{0}}{2}\right)-1-\alpha(d-1)\right\}
$$

then we will have

$$
\mathbf{E}\left[A_{L}\right]=O\left(L^{\alpha-1} \ln L\right)=o(1)
$$

and

$$
\mathbf{E}\left[B_{L}^{s_{0} / 2}\right]=O\left(L^{-\tilde{\beta}} \ln L\right)=o(1)
$$

with

$$
\tilde{\beta}=d\left(2-\frac{s_{0}}{2}\right)-1-\alpha(d-1)-m \beta>0 .
$$

In particular, we have

$$
A_{L}+B_{L} \rightarrow 0 \text { (in probability), }
$$

uniformly in $z \in\{|z-E|<r\} \cap \mathbf{C}_{+}$. From this and (2.33), it is easily seen that in order to prove our theorem, it is sufficient to prove

$$
\lim _{L \rightarrow \infty} \mathbf{E}\left[\exp \left\{-\frac{1}{|\Lambda|} \sum_{p} \sum_{j=1}^{n} a_{j} \Im \operatorname{Tr} G^{C_{p}}\left(E+|\Lambda|^{-1} \zeta_{j}\right)\right\}\right]=\mathscr{L}_{P}(\varphi)
$$

instead of (2.25).

Now let $E_{j}\left(C_{p}\right)$ be the eigenvalues of $H^{C_{p}}$, and consider the point process $\eta\left(C_{p} ; E\right), 1 \leqq p \leqq N_{L}^{d}$, defined by $(1.22)$, and let $\eta(L ; E)$ be their superposition as in (1.23). Then we can rewrite (2.42) as

$$
\lim _{L \rightarrow \infty} \mathbf{E}[\exp \{-\eta(L ; E)(\varphi)\}]=\mathscr{L}_{P}(\varphi)
$$

with $\varphi$ running through $\mathscr{A}$. Since, as before, we can show

$$
\mathbf{E}\left[\eta\left(C_{p} ; E\right)(d x)\right] \leqq N_{L}^{-d}\|\rho\|_{\infty} d x
$$

and

$$
\mathbf{E}[\eta(L ; E)(d x)] \leqq\|\rho\|_{\infty} d x,
$$

Lemma 1 and (2.43) tell us that our theorem is a direct consequence of the following

Proposition. As $L \rightarrow \infty, \eta(L ; E)$ converges weakly to the Poisson point process with the intensity measure $n(E) d x$.

Step 4. We turn now to the proof of the proposition. It is clear that $\eta\left(C_{p} ; E\right)$ are independent for different $p$, and (2.44) shows they constitute a uniformly asymptotically negligible array in the sense that for all bounded Borel sets $A$,

$$
\lim _{L \rightarrow \infty} \sup _{1 \leqq p \leqq N_{L}^{d}} \mathbf{P}\left(\eta\left(C_{p} ; E\right)(A)>0\right)=0
$$

(see Sect. 9.2 of $[\mathrm{D}-\mathrm{V}]$ ). Hence by Theorem 9.2.V. of [D-V], to prove that their superposition $\eta\left(C_{p} ; E\right)$ converges weakly to the Poisson point process with intensity measure $n(E) d x$, it suffices to show that (1.24) and (1.25) hold for any bounded interval $A \subset \mathbf{R}$. For this purpose, it is enough to show for any $\zeta=\sigma+i \tau \in \mathbf{C}_{+}$,

$$
N_{L}^{d} \mathbf{E}\left[\eta\left(C_{p} ; E\right)\left(f_{\zeta}\right)\right] \rightarrow \pi n(E)
$$


and

$$
\mathbf{E}\left[\sum_{i \neq j} f_{\zeta}\left(y_{i}\right) f_{\zeta}\left(y_{j}\right)\right]=o\left(N_{L}^{-d}\right)
$$

uniformly in $p$, where $f_{\zeta}$ was defined in (2.17) and we have set

$$
y_{j}=|\Lambda|\left(E_{j}\left(C_{p}\right)-E\right) .
$$

In fact, if we note $(2.44)$ and the fact that $1_{A}$ is approximated by elements in $\mathscr{A}$, it is easy to conclude from (2.47) that

$$
N_{L}^{d} \mathbf{E}\left[\eta\left(C_{p} ; E\right)(A)\right] \rightarrow n(E)|A| .
$$

Moreover for any finite interval $A$, we can choose $\zeta \in \mathbf{C}_{+}$and $a>0$ so that

$$
1_{A} \leqq a f_{\zeta}
$$

holds. Hence (2.48) implies

$$
\mathbf{E}\left[\sum_{i \neq j} 1_{A}\left(y_{i}\right) 1_{A}\left(y_{j}\right)\right]=o\left(N_{L}^{-d}\right) .
$$

To show that (2.50) and (2.52) imply (1.24) and (1.25), note that

$$
\begin{aligned}
\sum_{j \geqq 2} \mathbf{P}\left(\eta\left(C_{p} ; E\right)(A) \geqq j\right) & =\sum_{j=2}^{\infty}(j-1) \mathbf{P}\left(\eta\left(C_{p} ; E\right)(A)=j\right) \\
& \leqq \sum_{j=2}^{\infty} j(j-1) \mathbf{P}\left(\eta\left(C_{p} ; E\right)(A)=j\right) \\
& =\mathbf{E}\left[\eta\left(C_{p} ; E\right)(A)\left\{\eta\left(C_{p} ; E\right)(A)-1\right\}\right] \\
& =\mathbf{E}\left[\sum_{i \neq j} 1_{A}\left(y_{i}\right) 1_{A}\left(y_{j}\right)\right]=o\left(N_{L}^{-d}\right),
\end{aligned}
$$

uniformly in $p$, which yields (1.25) at once. On the other hand, (1.24) is a direct consequence of (2.50), (2.53) and

$$
\mathbf{P}\left(\eta\left(C_{p} ; E\right) \geqq 1\right)=\mathbf{E}\left[\eta\left(C_{p} ; E\right)(A)\right]-\sum_{j \geqq 2} \mathbf{P}\left(\eta\left(C_{p} ; E\right)(A) \geqq j\right) .
$$

Step 5. Let us prove (2.47). As before, we have

$$
\begin{aligned}
\mathbf{E}\left[\eta\left(C_{p} ; E\right)\left(f_{\zeta}\right)\right] & =\frac{1}{|\Lambda|} \mathbf{E}\left[\Im \operatorname{Tr} G^{C_{p}}(\lambda)\right] \\
& =\frac{1}{|\Lambda|} \mathbf{E}\left[\left\{\sum_{x \in \operatorname{int}\left(C_{p}\right)}+\sum_{x \in C_{p} \backslash \operatorname{int}\left(C_{p}\right)}\right\} \Im G^{C_{p}}(\lambda ; x, x)\right],
\end{aligned}
$$

where $\lambda=E+|\Lambda|^{-1} \zeta$. In the same way as we estimated $\mathbf{E}\left[A_{L}\right]$ in Step 3, we can estimate the second term on the right-hand side of (2.55) to be of

$$
O\left(L^{-d}\left(L / N_{L}\right)^{d-1} l_{L}\right)=o\left(N_{L}^{-d}\right)
$$

if we choose $N_{L}$ and $l_{L}$ as in Step 3. 
On the other hand, for $x \in \operatorname{int}\left(C_{p}\right)$ and $z \in \mathbf{C}_{+}$, we have

$$
G^{C_{p}}(z ; x, x)=G(z ; x, x)-\sum_{\left\langle y, y^{\prime}\right\rangle \in \partial C_{p}} G^{C_{p}}(z ; x, y) G\left(z ; y^{\prime}, x\right) .
$$

Hence

$$
\begin{aligned}
&\left|\mathbf{E}\left[\Im G^{C_{p}}(\lambda ; x, x)\right]-\mathbf{E}[\Im G(\lambda ; x, x)]\right| \\
& \quad \leqq \sum_{\left\langle y, y^{\prime}\right\rangle \in \partial C_{p}} \mathbf{E}\left[\left|G^{C_{p}}(\lambda ; x, y) G\left(\lambda ; y^{\prime}, x\right)\right|\right] \\
& \quad \leqq(|\Lambda| / \tau)^{2-s_{0}} \sum_{\left\langle y, y^{\prime}\right\rangle \in \partial C_{p}} \mathbf{E}\left[\left|G^{C_{p}}(\lambda ; x, y) G\left(\lambda ; y^{\prime} x\right)\right|^{s_{0} / 2}\right] \\
& \leqq(|\Lambda| / \tau)^{2-s_{0}} \sum_{\left\langle y, y^{\prime}\right\rangle \in \partial C_{p}} \sqrt{\mathbf{E}\left[\left|G^{C_{p}}(\lambda ; x, y)\right|^{s_{0}}\right]} \sqrt{\mathbf{E}\left[\left|G\left(\lambda ; y^{\prime}, x\right)\right|^{s_{0}}\right]} \\
&=O\left(L^{\left(3-s_{0}\right) d-1} N_{L}^{-d+1} e^{-m l_{L} / 2}\right) .
\end{aligned}
$$

In the above calculation, we used the condition (1.20) and the fact that

$$
\left|G^{C_{p}}(z ; x, y)\right| \leqq(\Im z)^{-1} ; \quad|G(z ; x, y)| \leqq(\Im z)^{-1}
$$

and that $\mathbf{E}\left|G\left(z ; y^{\prime} x\right)\right|^{s_{0}}$ is bounded by a constant independent of $y^{\prime}, x \in \mathbf{Z}^{d}$ and $z \in \mathbf{C}_{+}$. Now let us take $l_{L}=\beta^{\prime} \ln L$ with $\beta^{\prime}$ large enough. Then we will have

$$
\mathbf{E}\left[\Im G^{C_{p}}(\lambda ; x, x)\right]=\mathbf{E}[\Im G(\lambda ; x, x)]+o(1)
$$

as $L \rightarrow \infty$, where $o(1)$ is uniform in $x \in \mathbf{Z}^{d}$. But as is well known, for all $x \in \mathbf{Z}^{d}$,

$$
\mathbf{E}[\Im G(\lambda ; x, x)]=\Im \int_{-\infty}^{\infty} \frac{1}{u-\lambda} d N(u)
$$

If $N(u)$ is differentiable at $u=E$, then the right-hand side converges to

$$
\pi n(E)=\left.\pi \frac{d N(u)}{d u}\right|_{u=E}
$$

as $L \rightarrow \infty$, since $\lambda \rightarrow E$ non-tangentially (see Chapter IV of [D]). Combining these estimates with (2.55), we arrive at

$$
\mathbf{E}\left[\eta\left(C_{p} ; E\right)\left(f_{\zeta}\right)\right] \sim \frac{1}{|\Lambda|}\left|\operatorname{int}\left(C_{p}\right)\right| \pi n(E) \sim \pi N_{L}^{-d} n(E),
$$

as $L \rightarrow \infty$.

Step 6. To finish the proof of the theorem, we shall now prove (2.48). In view of (2.49), the left-hand side of (2.48) multiplied by $|\Lambda|^{2}$ can be rewritten as

$$
\begin{aligned}
\mathbf{E} & {\left[\sum_{i \neq j} \Im \frac{1}{E_{i}\left(C_{p}\right)-\lambda} \Im \frac{1}{E_{j}\left(C_{p}\right)-\lambda}\right] } \\
& =\mathbf{E}\left[\left\{\operatorname{Tr} \Im G^{C_{p}}(\lambda)\right\}^{2}-\operatorname{Tr}\left\{\left(\Im G^{C_{p}}(\lambda)\right)^{2}\right\}\right]
\end{aligned}
$$




$$
\begin{aligned}
& =\mathbf{E}\left[\sum_{x, y \in C_{p}}\left\{\Im G^{C_{p}}(\lambda ; x, x) \Im G^{C_{p}}(\lambda ; y, y)-\Im G^{C_{p}}(\lambda ; x, y) \Im G^{C_{p}}(\lambda ; y, x)\right\}\right] \\
& =\mathbf{E}\left[\sum_{x, y \in C_{p}} \operatorname{det}\left(\begin{array}{cc}
\Im G^{C_{p}}(\lambda ; x, x) & \Im G^{C_{p}}(\lambda ; x, y) \\
\Im G^{C_{p}}(\lambda ; x, y) & \Im G^{C_{p}}(\lambda ; y, y)
\end{array}\right)\right]
\end{aligned}
$$

where as before $\lambda=E+|\Lambda|^{-1} \zeta$. Since $|\Lambda|=L^{d}$ and $\left|C_{p}\right| \sim\left(L / N_{L}\right)^{d}$ uniformly in $p,(2.48)$ is an immediate consequence of the following

Lemma 2. For any $z \in \mathbf{C}_{+}, D \subset \mathbf{Z}^{d}$, and $x, y \in D$ with $x \neq y$, one has

$$
\mathbf{E}\left[\operatorname{det}\left(\begin{array}{cc}
\Im G^{D}(z ; x, x) & \Im G^{D}(z ; x, y) \\
\Im G^{D}(z ; y, x) & \Im G^{D}(z ; y, y)
\end{array}\right)\right] \leqq \pi^{2}\|\rho\|_{\infty}^{2} .
$$

Proof. Set

$$
g(z)=\left(\begin{array}{ll}
G^{D}(z ; x, x) & G^{D}(z ; x, y) \\
G^{D}(z ; y, x) & G^{D}(z ; y, y)
\end{array}\right)
$$

If we define $\tilde{V}=V-V_{x} \delta_{x}-V_{y} \delta_{y}$ and if we construct from this $\tilde{V}$,

$$
\tilde{H}^{D}=-\Delta^{D}+\tilde{V} ; \quad \tilde{G}^{D}(z)=\left(\tilde{H}^{D}-z\right)^{-1}
$$

and

$$
\tilde{g}(z)=\left(\begin{array}{cc}
\tilde{G}^{D}(z ; x, x) & \tilde{G}^{D}(z ; x, y) \\
\tilde{G}^{D}(z ; x, y) & \tilde{G}^{D}(z ; y, y)
\end{array}\right),
$$

then by Krein's formula (see Appendix I of $[\mathrm{A}-\mathrm{M}]$ ), we obtain

$$
g(z)=\left[\left(\begin{array}{cc}
V_{x} & 0 \\
0 & V_{y}
\end{array}\right)+\tilde{g}(z)^{-1}\right]^{-1} .
$$

Now for any invertible complex matrix $A$, we have in general

$$
A^{-1}-\bar{A}^{-1}=A^{-1}(\bar{A}-A) \bar{A}^{-1}=\bar{A}^{-1}(\bar{A}-A) A^{-1},
$$

where $\bar{A}$ is the complex conjugate of $A$. Using this formula twice, we can compute

$$
\begin{aligned}
2 i \Im g(z) & =g(z)-g(\bar{z}) \\
& =\left[v+\tilde{g}(z)^{-1}\right]^{-1}\left[\tilde{g}(\bar{z})^{-1}-\tilde{g}(z)^{-1}\right]\left[v+\tilde{g}(\bar{z})^{-1}\right]^{-1} \\
& =\left[v+\tilde{g}(z)^{-1}\right]^{-1} \tilde{g}(z)^{-1}[\tilde{g}(z)-\tilde{g}(\bar{z})] \tilde{g}(\bar{z})^{-1}\left[v+\tilde{g}(\bar{z})^{-1}\right]^{-1},
\end{aligned}
$$

so that

$$
\operatorname{det} \Im g(z)=(\operatorname{det} \Im \tilde{g}(z))|\operatorname{det} \tilde{g}(z)|^{-2}\left|\operatorname{det}\left(v+\tilde{g}(z)^{-1}\right)\right|^{-2},
$$

where we have set

$$
v=\left(\begin{array}{cc}
V_{x} & 0 \\
0 & V_{y}
\end{array}\right)
$$


We shall now prove

$$
\begin{aligned}
I & =\int_{-\infty}^{\infty} \int_{-\infty}^{\infty}\left|\operatorname{det}\left(v+\tilde{g}(z)^{-1}\right)\right|^{-2} d V_{x} d V_{y} \\
& =\pi^{2}|\operatorname{det} \tilde{g}(z)|^{2}\left\{\operatorname{det} \Im \tilde{g}(z)\left(\operatorname{det} \Im \tilde{g}(z)+\left|\tilde{G}^{D}(z ; x, y)\right|^{2}\right)\right\}^{-1 / 2},
\end{aligned}
$$

which gives (2.65) because of (2.72) and condition (1.4-5). To prove (2.74), we let

$$
\tilde{g}(z)^{-1}=\left(\begin{array}{ll}
a & b \\
b & c
\end{array}\right)=\left(\begin{array}{ll}
a_{1}+i a_{2} & b_{1}+i b_{2} \\
b_{1}+i b_{2} & c_{1}+i c_{2}
\end{array}\right) .
$$

Note that this matrix depends neither on $V_{x}$ nor $V_{y}$. Then

$$
\left|\operatorname{det}\left(v+\tilde{g}(z)^{-1}\right)\right|^{-2}=\left|\left(V_{x}+a\right)\left(V_{y}+c\right)-b^{2}\right|^{-2},
$$

and by shifting the variable of integration, the right-hand side of (2.74) becomes

$$
\begin{aligned}
I & =\int_{-\infty}^{\infty} \int_{-\infty}^{\infty}\left|\left(V_{x}+i a_{2}\right)\left(V_{y}+i c_{2}\right)-b^{2}\right|^{-2} d V_{x} d V_{y} \\
& =\int_{-\infty}^{\infty} \frac{d V_{x}}{\left|V_{x}+i a_{2}\right|^{2}} \int_{-\infty}^{\infty} \frac{d V_{y}}{\left|V_{y}+\left(i c_{2}-\frac{b^{2}}{V_{x}+i a_{2}}\right)\right|^{2}}
\end{aligned}
$$

Now if $\Im \zeta \neq 0$, we have

$$
\int_{-\infty}^{\infty} \frac{d t}{|t+\zeta|^{2}}=\frac{\pi}{|\Im \zeta|}
$$

We will apply this formula to compute the integral (2.77). For this purpose, first note that by $(2.70)$,

$$
\begin{aligned}
\left(\begin{array}{ll}
a_{2} & b_{2} \\
b_{2} & c_{2}
\end{array}\right) & =\frac{1}{2 i}\left\{\tilde{g}(z)^{-1}-\tilde{g}(\bar{z})^{-1}\right\}=\frac{1}{2 i} \tilde{g}(z)^{-1}\{\tilde{g}(\bar{z})-\tilde{g}(z)\} \tilde{g}(\bar{z})^{-1} \\
& =-\tilde{g}(z)^{-1}(\Im \tilde{g}(z)) \tilde{g}(\bar{z})^{-1}
\end{aligned}
$$

Since $\Im \tilde{g}(z)$ is a positive definite matrix, we have

$$
\operatorname{det}\left(\begin{array}{ll}
a_{2} & b_{2} \\
b_{2} & c_{2}
\end{array}\right)=|\operatorname{det} \tilde{g}(z)|^{-2} \operatorname{det}(\Im \tilde{g}(z))>0 .
$$

In particular, we have $a_{2} \neq 0$ and $c_{2} \neq 0$. Hence we can compute without difficulty

$$
\begin{aligned}
\Im\left(i c_{2}-\frac{b^{2}}{V_{x}+i a_{2}}\right) & =\frac{1}{V_{x}^{2}+a_{2}^{2}}\left\{c_{2}\left(V_{x}^{2}+a_{2}^{2}\right)-2 b_{1} b_{2} V_{x}+a_{2}\left(b_{1}^{2}-b_{2}^{2}\right)\right\} \\
& =\frac{1}{V_{x}^{2}+a_{2}^{2}}\left\{c_{2}\left(V_{x}-\frac{b_{1} b_{2}}{c_{2}}\right)^{2}+\frac{1}{c_{2}} \operatorname{det} h\left(|b|^{2}+\operatorname{det} h\right)\right\} \\
& >0
\end{aligned}
$$


where we have set for brevity

$$
h=\left(\begin{array}{ll}
a_{2} & b_{2} \\
b_{2} & c_{2}
\end{array}\right),
$$

so that by using (2.78) twice, the right-hand side of (2.77) is computed to give

$$
\begin{gathered}
\pi \int d V_{x}\left\{c_{2}\left(V_{x}-\frac{b_{1} b_{2}}{c_{2}}\right)^{2}+\frac{1}{c_{2}} \operatorname{det} h\left(|b|^{2}+\operatorname{det} h\right)\right\}^{-1} \\
=\pi^{2}\left\{\operatorname{det} h\left(|b|^{2}+\operatorname{det} h\right)\right\}^{-1 / 2} .
\end{gathered}
$$

Equation (2.74) now follows at once if we substitute (2.80), (2.82) and

$$
b=-(\operatorname{det} \tilde{g}(z))^{-1} \tilde{G}^{D}(z ; x, y) .
$$

The proof of Lemma 2, and hence of our theorem is now complete.

\section{Appendix. On the Condition (1.20) in the One-Dimensional Case}

In this appendix, we shall prove the following proposition announced in the remark after the statement of the theorem.

Proposition A.1. In the case of $d=1$, if we suppose

$$
\int|v|^{\varepsilon} \rho(v) d v<\infty \text { for some } \varepsilon>0
$$

in addition to (1.4-5), then condition (1.20) is true for all $E \in \mathbf{R}$.

Proof. We will use some techniques in [C-K-M], [v-K] and [K-L-S] by "complexifying" them.

Let $\Lambda=[a, b], a \leqq x<b$ and $y=b \in \partial \Lambda$. By the resolvent equation, we get

$$
G^{[a, b]}(z ; x, b)=G^{[x, b]}(z ; x, b)\left\{1+G^{[a, b]}(z ; x, x-1)\right\}
$$

for all $z \in \mathbf{C}_{+}$. Since we already know that $\mathbf{E}\left|G^{\Lambda}(z ; x, y)\right|^{s}$ is bounded uniformly in $\Lambda, x, y \in \Lambda$ and $z \in \mathbf{C}_{+}$for each $s \in(0,1)$, it suffices to prove the exponential decay of $\mathbf{E}\left|G^{[x, b]}(z ; x, b)\right|^{s}$ for some $s \in(0,1)$.

To this end, consider

$$
\tilde{H}^{[x, b]}=H^{[x, b]}-i P_{b},
$$

where $P_{b}$ is the projection onto the one-dimensional subspace of $l^{2}\left(\mathbf{Z}^{1}\right)$ spanned by $\delta_{b}$. Then for any $z \in \mathbf{C}_{+}, \tilde{H}^{[x, b]}-z$ is invertible. To see this, suppose $\left(\tilde{H}^{[x, b]}-z\right) \varphi=0$ with $\varphi=\varphi(x)$ vanishing outside of $[x, b]$. Then $(H-z) \varphi(t)=0$ for $t \in[x, b-1]$. Hence we have $\varphi(t)=\varphi(x) \psi(t)$ for $t \in[x, b]$, where $\psi$ is the solution of $H \psi=z \psi$ satisfying $\psi(x-1)=0$ and $\psi(x)=1$. Suppose $\varphi(x) \neq 0$, then we would have from this $\left(\tilde{H}^{[x, b]}-z\right)\left(\psi 1_{[x, b]}\right)=0$, in particular

$$
-\psi(b-1)+\left(V_{b}-z\right) \psi(b)-i \psi(b)=0 .
$$

But since $(H-z) \psi(b)=0$, this leads to

$$
\psi(b+1)-i \psi(b)=0 .
$$


In view of Lemma A.2 below, (A.5) shows $\psi(t) \equiv 0$, which is a contradiction, and hence we must have $\varphi(x)=0$, namely $\varphi(t) \equiv 0$.

Lemma A.2. For any $y>x$, we have

$$
|\psi(y+1)-i \psi(y)|^{2}=|\psi(y+1)|^{2}+|\psi(y)|+\Im z \sum_{j=x}^{y}|\psi(j)|^{2} .
$$

Now we can consider $\tilde{G}^{[x, b]}(z)=\left(\tilde{H}^{[x, b]}-z\right)^{-1}$. Then we have

$$
\tilde{G}^{[x, b]}(z ; x, b)=\{\psi(b+1)-i \psi(b)\}^{-1} .
$$

In fact, if we set

$$
u(t)=\tilde{G}^{[x, b]}(z ; t, b)=\left(\tilde{G}^{[x, b]}(z) \delta_{b}\right)(t),
$$

then we must have $(H u)(t)=0$ for $t \in[x, b-1]$. Hence $u(t)=u(x) \psi(t)$ for $t \in[x, b]$. But

$$
\begin{aligned}
1 & =\left(\left(\tilde{H}^{[x, b]}-z\right) u\right)(b)=-u(b-1)+\left(V_{b}-z\right) u(b)-i u(b) \\
& =u(x)\left\{-\psi(b-1)+\left(V_{b}-z\right) \psi(b)-i \psi(b)\right\} \\
& =\tilde{G}^{[x, b]}(z ; x, b)\{\psi(b+1)-i \psi(b)\}
\end{aligned}
$$

as desired.

On the other hand, again by the resolvent equation,

$$
\begin{aligned}
G^{[x, b]}(z ; x, b) & =\tilde{G}^{[x, b]}(z ; x, b)-i\left(\tilde{G}^{[x, b]}(z) P_{b} G^{[x, b]}(z)\right)(x, b) \\
& =\tilde{G}^{[x, b]}(z ; x, b)\left[1-i G^{[x, b]}(z ; b, b)\right] .
\end{aligned}
$$

Again since

$$
\mathbf{E}\left|G^{[x, b]}(z ; b, b)\right|^{s}
$$

is bounded for any $s \in(0,1)$, we only need to prove the exponential decay of

$$
\mathbf{E}\left|\tilde{G}^{[x, b]}(z ; x, b)\right|^{s}
$$

for some $s \in(0,1)$. But since we have by (A.7) and Lemma A.2,

$$
\left|\tilde{G}^{[x, b]}(z ; x, b)\right| \leqq\left\|\left(\begin{array}{c}
\psi(b+1) \\
\psi(b)
\end{array}\right)\right\|^{-1},
$$

and since Lemma 5.1 of $[\mathrm{C}-\mathrm{K}-\mathrm{M}]$ is still valid for complex parameter $E$, we are done.

Proof of Lemma A.2. Substitute

$$
\begin{aligned}
& \psi(y+1) \bar{\psi}(y)-\bar{\psi}(y+1) \psi(y) \\
& \quad=\bar{\psi}(y)\left\{-\psi(y-1)+\left(V_{y}-z\right) \psi(y)\right\}-\psi(y)\left\{-\bar{\psi}(y-1)+\left(V_{y}-\bar{z}\right) \bar{\psi}(y)\right\} \\
& =\{\psi(y) \bar{\psi}(y-1)-\bar{\psi}(y) \psi(y-1)\}-2 i(\Im z)|\psi(y)|^{2} \\
& \quad=\cdots=-2 i(\Im z) \sum_{j=x}^{y}|\psi(j)|^{2}
\end{aligned}
$$


into

$$
\begin{aligned}
& |\psi(y+1)-i \psi(y)|^{2} \\
& \quad=\{\psi(y+1)-i \psi(y)\}\{\bar{\psi}(y+1)+i \bar{\psi}(y)\} \\
& \quad=|\psi(y+1)|^{2}+|\psi(y)|^{2}+i\{\psi(y+1) \bar{\psi}(y)-\bar{\psi}(y+1) \psi(y)\} .
\end{aligned}
$$

Acknowledgement. The author thanks Professors R. Carmona, L. Xu, A. Klein and S. Jitomirskaya and the staff of the Department of Mathematics, University of California at Irvine, where this work was done, for their warm hospitality and encouragement.

\section{References}

[A-M] Aizenman, M., Molchanov, S.A.: Localization at large disorder and at extreme energies. Commun. Math. Phys. 157, 245-278 (1993)

[C-K-M] Carmona, R., Klein, A., Martinelli, F.: Anderson localization for Bernoulli and other singular potentials. Commun. Math. Phys. 108, 41-66 (1987)

[C-L] Carmona, R., Lacroix, J.: Spectral theory of random Schrödinger operators. Boston: Birkhäuser, 1990

[D-V] Daley, D.J., Vere-Jones, D.: An introduction to the theory of point processes. Berlin, Heidelberg New York: Springer, 1988

[D-L-S] Delyon, F., Lévy, Y., Souillard, B.: Anderson localization for multi-dimensional systems at large disorder or large energy. Commun. Math. Phys. 100, 463-470 (1985)

[D] Donoghue, W.F.: Monotone matrix functions and analytic continuation. Berlin, Heidelberg, New York: Springer, 1974

[G-M-P] Goldsheidt, I.Ya., Molchanov, S.A., Pastur, L.A.: A pure point spectrum of the stochastic one-dimensional Schrödinger operator. Funct. Anal. Appl. 11, 1-11 (1977)

[G] Graf, G.M.: Anderson localization and the space-time characteristic of continuum states. J. Stat. Phys. 75, 337-346 (1994)

[K-L-S] Klein, A., Lacroix, J., Speis, A.: Localization for the Anderson model on a strip with singular potentials. J. Funct. Anal. 94, 135-155 (1990)

[Mi] Minami, N.: Level clustering in a one-dimensional finite system. Prog. Theor. Phys. Suppl. 116, 359-368 (1994)

[Mo1] Molchanov, S.A.: The structure of eigenfunctions of one-dimensional unordered structure. Math. USSR, Izv. 12, 69-101 (1978)

[Mo2] Molchanov, S.A.: The local structure of the spectrum of the one-dimensional Schrödinger operator. Commun. Math. Phys. 78, 429-446 (1981)

[S] Spencer, T.: The Schrödinger operator with a random potential. In: Osterwalder, K., Stora, R. (eds.) Critical phenomena, random system, gauge theories. Les Houches, Session XLIII, 1984, Amsterdam: North-Holland, 1986, pp. 895-943

[v-K] von Dreifus, H., Klein, A.: A new proof of localization in the Anderson tight binding model. Commun. Math. Phys. 124, 285-299 (1989) 
